\title{
Relevamiento herpetológico de los municipios de Avellaneda y Quilmes (Buenos Aires, Argentina)
}

\author{
Herpetological Survey of Avellaneda and Quilmes Counties \\ (Buenos Aires, Argentina)
}

Lucas Lerzo', Gonzalo Reuter', David Gustavo Vera², Felipe Andrés Suazo Lara², Ana Belén Tenorio', Isabel Ferreyra1, Elián Leandro Guerrero ${ }^{*}$

1 Facultad de Ciencias Naturales y Museo, Universidad Nacional de La Plata. Calle 60 y 120, (B1900) La Plata, Buenos Aires.

2 División Zoología Vertebrados. Museo de La Plata. Facultad de Ciencias Naturales y Museo, Universidad Nacional de La Plata. Paseo del Bosque s/n., (B1900) La Plata, Buenos Aires. CONICET.

3 Red Paleontológica U-Chile, Laboratorio de Ontogenia y Filogenia, Departamento de Biología, Facultad de Ciencias, Universidad de Chile, Av. Las Palmeras 3425, (7800003) Santiago, Chile.

4 División Plantas Vasculares. Museo de La Plata. Facultad de Ciencias Naturales y Museo, Universidad Nacional de La Plata. Paseo del Bosque s/n., (B1900) La Plata, Buenos Aires. CONICET.

* Autor para correspondencia: eguerrero@fcnym.unlp.edu.ar

\section{RESUMEN}

El objetivo del presente estudio es realizar un inventario de anfibios y reptiles de los partidos de Avellaneda y Quilmes, un sector suburbano de la costa nordeste de la provincia de Buenos Aires, Argentina. El área se visitó esporádicamente entre 2005 y 2014 con muestreos ocasionales, y con visitas sistematizadas entre 2014 y 2017. Se dividió el área de estudio en cuatro unidades ambientales mayores (1-playa; 2-bosque y bañados; 3-barranca; y 4-terraza alta) anotando en cuál de ellos fue hallada cada especie. Se encontraron 33 especies (13 especies de anfibios y 20 especies de reptiles), la mayoría de ellos en los bosques y bañados. La totalidad de las especies de anfibios son nativas, mientras que entre los reptiles se cuenta con dos especies exóticas. Los anfibios y reptiles del área costera de Quilmes y Avellaneda exponen la vinculación faunística entre las selvas y humedales de la provincia Paranaense con el Río de la Plata. Finalmente, se destaca la poca representación de especies de pastizal que se

\footnotetext{
Ref. bibliográfica: Lerzo, L.; Reuter, G.; Vera, D. G.; Suazo Lara, F. A.; Tenorio, A. B.; Ferreyra, I.; Guerrero, E. L. 2019. "Relevamiento herpetológico de los municipios de Avellaneda y Quilmes (Buenos Aires, Argentina)". Acta zoológica lilloana 63 (2): 68-82. Fundación Miguel Lillo, Tucumán, Argentina. D.O.I.: https://doi. org/10.30550/j.azl/2019.63.2/5

> Recibido: 22/04/19 - Aceptado: 04/11/19

$>$ URL de la revista: http://actazoologica.lillo.org.ar

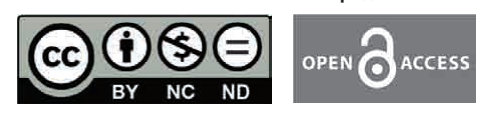

> Algunos derechos reservados. Esta obra está bajo una Licencia Creative Commons Atribución - No Comercial - Sin Obra Derivada 4.0 Internacional.
} 
han registrado en áreas cercanas, pero no en el área de estudio. Probablemente, esto se deba a que las actividades antrópicas en la región afectaron irreversiblemente a los pastizales originales.

Palabras clave - Dispersión pasiva, pastizales pampeanos, perturbación antrópica, provincia Paranaense, Río de la Plata.

\section{ABSTRACT}

The objective of this study is to perform an inventory of amphibians and reptiles of Avellaneda and Quilmes districts, a suburban sector of the northeast coast of the province of Buenos Aires, Argentina. The area was visited sporadically between 2005 and 2014 with occasional sampling, and with systematized visits between 2014 and 2017. The study area was divided into four major environmental units (1-beach, 2forest and wetlands, 3-slope, and 4- high terrace) noting in which of them was found each species. 33 species were found (13 amphibian species and 20 reptile species), most of them in forests and wetlands. All of the amphibians are native, while among the reptiles there are two exotic species. The amphibians and reptiles of the coastal area of Quilmes and Avellaneda expose the faunal link between the forests and the wetlands of the Paranaense province with the Río de la Plata. Finally, highlight out the low representation of grassland species which have been recorded in nearby areas but not in the study area. Probably, this is because the anthropic activities in the region irreversibly affected the original grasslands.

Keywords - Passive dispersal, Pampean Grasslands, anthropogenic disturbance, Paranaense province, Río de la Plata.

\section{INTRODUCCIÓN}

La fauna de anfibios y reptiles de la provincia de Buenos Aires ha sido estudiada por Barrio (1962), Gallardo (1974; 1977; 1980; 1987) y Williams (1991), entre otros. Se han estudiado en detalle las Sierras de los sistemas de Ventania (Koslowsky, 1896; Di Pietro, 2016) y de Tandilia (Vega y Bellagamba, 1990) en el centro-sur de la provincia, la Reserva Natural Otamendi (Pereira y Haene 2003) en el norte, los partidos del noroeste (Agostini, Saibene, Roesler, Bilenca, 2016), y la Costa Atlántica (Kacoliris, Horlent, Williams, 2006) en el este. La costa del Río de la Plata en el nordeste de la provincia de Buenos Aires se encuentra relativamente bien estudiada, aunque los trabajos existentes se enfocan al sector entre Punta Lara y Bahía Samborombón. Monitoreos recientes en la Reserva Natural Punta Lara dieron cuenta de 16 especies de anfibios (Agostini, Saibene, Barraso, 2012) y 15 especies de reptiles (Saibene, Cajade, Pagano, Herrera, 2012). Esta reserva posee grandes extensiones de pajonales y algunos de los parches más australes de las selvas en galería del sistema fluvial (Cabrera y Dawson, 1944). Existen también inventarios de anfibios y reptiles del Parque Costero del Sur (Gallardo, 1987; Williams y Kacoliris, 2009). En la 
Ciudad Autónoma de Buenos Aires (CABA) se estima que habrían habitado en el pasado unas 16 especies de anfibios y 28 reptiles (Williams y Kacoliris, 2012). En la actualidad la composición faunística de esta ciudad y su conurbación se encuentra empobrecida, conociéndose casos documentados de extinciones locales de reptiles como Anisolepis undulatus (Wiegmann, 1834) (Saibene et al., 2012), anfibios como Melanophryniscus montevidensis (Philippi, 1902) (Agnolin y Guerrero, 2017), así como aves y plantas (Guerrero, Agnolin, Apodaca, 2018).

La región metropolitana de la CABA es una de las áreas de la República Argentina más afectadas por problemas ambientales resultantes de una urbanización poco planificada (Matteucci y Falcón, 2016; Roesler, Agostini, De Pino, Carricart, 2012). Las geoformas y la vegetación original de la costa del Río de la Plata fueron alteradas irreversiblemente por distintos tipos de contaminación o reducidas en superficie por la expansión urbana (Matteucci y Falcón, 2016). Algunas áreas naturales protegidas en el entorno de la CABA, como la Reserva Natural Ribera Norte, Reserva Ecológica Costanera Sur y la Reserva Natural de Punta Lara protegen especies y ambientes característicos de la Cuenca del Río de la Plata (Chebez, Gasparri, Athor, 2012). También se cuenta con otras áreas costeras de pretendida protección ambiental en varios municipios del conurbano sur. A pesar de poseer instrumento legal de protección, las reservas municipales Selva Marginal Quilmeña (Ordenanzas municipales 9348/02 y modificatoria $N^{\circ}$ 9508/03) y Los Sauces (Ordenanza Municipal 8255/96) en el partido de Quilmes y la Reserva Natural Selva Marginal de Hudson (Ordenanza Municipal 2.131 /91) en el partido de Berazategui, no poseen infraestructura ni personal comprometido. Sólo la primera de ellas fue revalorizada desde hace una década por la asamblea vecinal «No a la entrega de la costa», que ofrece visitas guiadas.

A partir del año 2010 se comenzó una serie de relevamientos periódicos de la diversidad de flora y fauna de la costa de los partidos de Avellaneda y Quilmes, ubicados entre la CABA y la Reserva Natural de Punta Lara. Se estudió la composición de especies de aves (Godoy, Suazo Lara, Guerrero, Rivero, González, Alegre, Godoy, Kain, Sesto, Chimento, 2012; Sosa, Rozadilla, Lazarte, Irazoqui, Montalibet, 2015), plantas (Guerrero, Suazo Lara, Chimento, Buet Constantino, Simón, 2012; Cabanillas, Barral, Guerrero, Chimento, 2016; Dosil Hiriart, Cabanillas, Apodaca, Benedictto, Barral, Guerrero, 2018), arácnidos (Guerrero et al., 2012); Montalibet et al., 2015; Rojas, Montalibet, Guerrero, 2016), miriápodos (Guerrero et al., 2012; Guerrero, 2013) y moluscos (Daglio, Suazo Lara, Lazarte, Lachowicz, 2014). Los inventarios biológicos son un paso fundamental para la planificación de estrategias de conservación tanto a nivel local como regional. También sirve para evaluar cuales son los ecosistemas y los elementos faunísticos más afectados por los cambios ambientales (Krebs, 2008). El objetivo de este trabajo es relevar la fauna de reptiles $\mathrm{y}$ anfibios de dicha área. 


\section{MATERIALES Y MÉTODOS}

\section{Área de estudio}

El área de estudio comprende la costa de los partidos de Avellaneda y Quilmes desde la calle Espora en el sudeste, hasta el Arroyo Santo Domingo al noroeste, entre las vías del Ferrocarril Roca y el Río de la Plata, abarcando 350 hectáreas de Avellaneda y 780 de Quilmes (Fig. 1). En esta área se incluye la Reserva Municipal Selva Marginal Quilmeña (RMSMQ) y la Reserva Natural Municipal Los Sauces, las barrancas de Bernal y Don Bosco, y parches de bañados y pastizales.

La RMSMQ cuenta con más de $150 \mathrm{Ha}$ de humedales y bosques típicos del norte de la provincia de Buenos Aires. Los bosques y lagunas de esta reserva se continúan por la costa hacia el noroeste en el partido de Avellaneda en terrenos no protegidos, pero igualmente valorados desde el punto de vista de la conservación de la biodiversidad (Guerrero et al., 2012; Cabanillas et al., 2016) y hacia el sureste hacia la Reserva Municipal Los Sauces, cuyos límites no son claros.

El área de estudio fue dividida en las siguientes categorías de ambientes (fig. 1): playa (playa de arena del Río de la Plata y juncales contiguos), bosque y bañados (bosques de la costa del Río de la Plata y humedales contiguos hasta la barranca), barranca (barranca de la zona urbana), y terraza alta (llanura por encima de la barranca, en la zona urbana). La playa y los bosques y bañados de la costa están separados del área urbana de la barranca y la terraza alta por el relleno sanitario (CEAMSE) y la autopista R. Balbín (Buenos Aires-La Plata). En el partido de Quilmes se encuentran todas las categorías ambientales mencionadas, mientras que en el sector estudiado del partido de Avellaneda no hay barranca ni terraza alta.

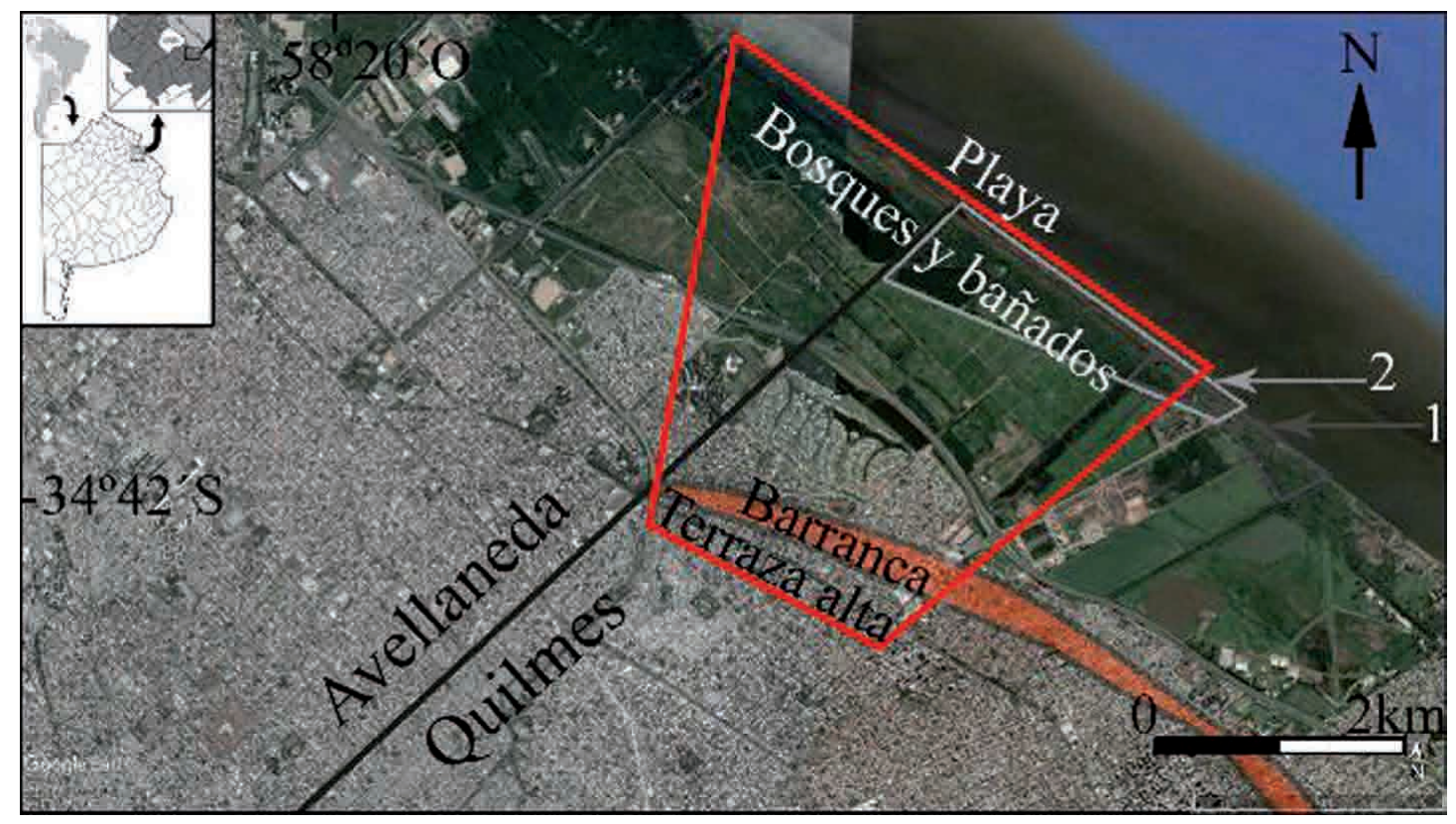

Figura 1. Mapa del área de estudio (recuadro rojo). 1, Reserva Municipal Los Sauces; 2, Reserva Municipal Selva Marginal Quilmeña. 


\section{Métodos de obtención de datos}

Se han realizado tareas de campo entre los años 2005 y 2018, en las cuales se relevaron las especies de anfibios y reptiles presentes en la zona. El área se recorrió irregularmente desde el año 2005 hasta el 2014 colectando ocasionalmente materiales por encuentro visual y búsqueda activa bajo troncos y piedras.

Desde el año 2014 hasta el año 2017 se desarrollaron muestreos sistematizados basándonos en los métodos estandarizados de muestreo de Heyer, Donnelly, Foster, Mcdiarmid (2014): En bosques y bañados se realizaron relevamientos por encuentros visuales en 5 transectas paralelas de 100 metros de longitud por 2 metros de ancho cada una, separadas entre sí por una distancia de 20 metros; relevamientos auditivos en todos los tipos de ambientes; y trampas de caída de 20 lts revisadas cada 5 días en ambientes de bosques y bañados. Acompañado a dichas actividades se levantaron rocas y troncos, se revisaron vegetales con hojas envainantes (e.g. Eryngium spp.) y se removieron camalotales. El esfuerzo de muestreo promedio fue de 5 personas en recorridos mensuales llevados a cabo entre las 10 y las 19 horas. $\mathrm{El}$ acceso al área de bosque y bañados es dificultoso debido a que no posee caminos públicos a pesar de emplazarse en un área suburbana, lo cual trajo numerosas limitaciones, entre las cuales se debe destacar la imposibilidad de trabajar en horario nocturno en la costa. Esto pudo haber introducido cierto sesgo en la detectabilidad de las especies.

En adición se estudiaron materiales colectados por alumnos de la escuela $\mathrm{N}^{\circ}$ 52 (Alicia Moreau de Justo, Avellaneda), supervisados por la Maestra L. N. Sancho tras un taller de herpetología dictado por E. L. Guerrero.

\section{Análisis de datos}

Para la asignación sistemática de cada especie se utilizó, en el caso de los Anfibios, el trabajo de Vaira et al., (2012). Para los Reptiles se utilizaron diferentes trabajos: en el caso de los Testudines se siguió el arreglo sistemático propuesto por Prado et al. (2012); para los escamados no ofidios se utilizó el trabajo de Abdala et al. (2012); y, por último, para las serpientes se utilizó el trabajo de Giraudo et al. (2012). Los ejemplares de referencia han sido depositados en el Museo de La Plata (MLP; ver apéndice).

\section{RESULTADOS}

Se registraron 13 especies de anfibios (tabla 1; 7 Hylidae, 4 Leptodactylidae y $2 \mathrm{Bu}$ fonidae) y 20 especies de reptiles (tabla 2; 1 Chelonidae, 2 Testudinidae, 1 Chelidae, 1 Amphisbaenidae, 1 Diploglossidae, 1 Gymnophthalmidae, 1 Teiidae, 1 Gekkonidae, 1 Leptotyphlopidae, 9 Dipsadidae y 1 Viperidae), totalizando 33 especies. Durante los muestreos realizados entre el año 2005 y 2014 se registraron 17 especies. Los muestreos sistematizados desde el 2014 hasta 2017 permitieron aumentar la cantidad de especies registradas. El área de bosques y bañados es la de mayor superficie y es la que mayor riqueza posee, ya que cuenta con 24 de las especies, seguido por el am- 
Tabla 1. Listado de especies de anfibios halladas en la costa de Avellaneda y Quilmes. $\mathrm{P}=$ playa; $\mathrm{BB}=$ bosques y bañados; $\mathrm{Br}=$ barranca; $\mathrm{T}=$ terraza alta.

\begin{tabular}{llc}
\hline Familia & Especie & Hallazgo \\
\hline Hylidae & Boana pulchella (Duméril \& Bibron, 1841) & BB, P \\
\hline & $\begin{array}{l}\text { Dendropsophus nanus (Boulenger, 1889) } \\
\text { Pseudis minuta (Günther, 1858) }\end{array}$ \\
\cline { 2 - 3 } & Pseudis limellum (Cope, 1862) & $\mathrm{B}, \mathrm{P}$ \\
\hline Scinax berthae (Barrio, 1962) & $\mathrm{BB}$ \\
\hline Scinax granulatus (Peters, 1871) & $\mathrm{BB}, \mathrm{P}$ \\
\hline Scinax squalirostris (Lutz, 1925) & $\mathrm{BB}$ \\
\hline Leptodactylidae & Leptodactylus gracilis (Duméril \& Bibron, 1841) \\
\hline & Leptodactylus latrans (Steffen, 1815) & $\mathrm{BB}, \mathrm{T}, \mathrm{P}$ \\
\hline Bufonidae & Reudopaludicola falcipes (Hensel, 1867) & $\mathrm{BB}, \mathrm{P}$ \\
\hline & Rhinella fernandezae (Gallardo, 1957) & $\mathrm{BB}, \mathrm{Br}, \mathrm{T}, \mathrm{P}$ \\
\hline
\end{tabular}

Tabla 2. Listado de especies de reptiles halladas en la costa de Avellaneda y Quilmes. $\mathrm{P}=$ playa; $\mathrm{BB}=$ bosques y bañados; $\mathrm{Br}=$ barranca; $\mathrm{T}=$ terraza alta.

\begin{tabular}{|c|c|c|c|}
\hline Orden & Familia & Especie & Hallazgo \\
\hline \multirow[t]{4}{*}{ Testudines } & Cheloniidae & Chelonia mydas (Linnaeus, 1758) & $P$ \\
\hline & Testudinidae & Chelonoidis chilensis (Gray, 1870) & $\mathrm{P}$ \\
\hline & Chelidae & Phrynops hilarii (Duméril \& Bibron, 1835) & BB \\
\hline & & Hydromedusa tectifera (Cope, 1869) & BB \\
\hline \multirow[t]{16}{*}{ Squamata } & Amphisbaenidae & Amphisbaena darwinii (Duméril \& Bibron, 1839) & $\mathrm{BB}, \mathrm{Br}$ \\
\hline & Diploglossidae & Ophiodes vertebralis (Bocourt, 1881) & BB \\
\hline & Gymnophthalmidae & Cercosaura schreibersii (Wiegmann, 1834) & BB \\
\hline & Teiidae & Salvator merianae (Duméril \& Bibron, 1839) & $\mathrm{BB}, \mathrm{Br}, \mathrm{P}$ \\
\hline & Gekkonidae & Tarentola mauritanica (Linnaeus, 1758) & $\mathrm{Br}$ \\
\hline & Leptotyphlopidae & Epictia albipuncta (Burmeister, 1861) & $\mathrm{Br}$ \\
\hline & Dipsadidae & Helicops infrataeniatus (Jan, 1865) & $\mathrm{P}$ \\
\hline & & Helicops leopardinus (Schlegel, 1837) & $\mathrm{P}$ \\
\hline & & Paraphimophis rusticus (Cope, 1878) & BB \\
\hline & & Thamnodynastes strigatus (Günther, 1858) & $P$ \\
\hline & & Thamnodynastes hypoconia (Cope, 1860) & $\mathrm{BB}, \mathrm{P}$ \\
\hline & & Xenodon dorbignyi (Bibron, 1854) & BB \\
\hline & & Philodryas patagoniensis (Girard, 1858) & BB \\
\hline & & Erythrolamprus semiaureus (Cope, 1862) & $\mathrm{BB}, \mathrm{P}$ \\
\hline & & Erythrolamprus poecilogyrus (Wied-neuwied, 1825) & BB \\
\hline & Viperidae & Bothrops alternatus (Duméril, Bibron \& Duméril, 1854) & $\mathrm{P}$ \\
\hline
\end{tabular}

biente de playa, que es la de menor superficie, que posee 16 especies. En este último ambiente, muchas de estas especies fueron recolectadas sobre camalotes (Guerrero et al. 2017). Las especies halladas en la barranca y la terraza alta son taxones que toleran la presencia humana, capaces de sobrevivir en terrenos baldíos, banquinas $\mathrm{y}$ jardines. 


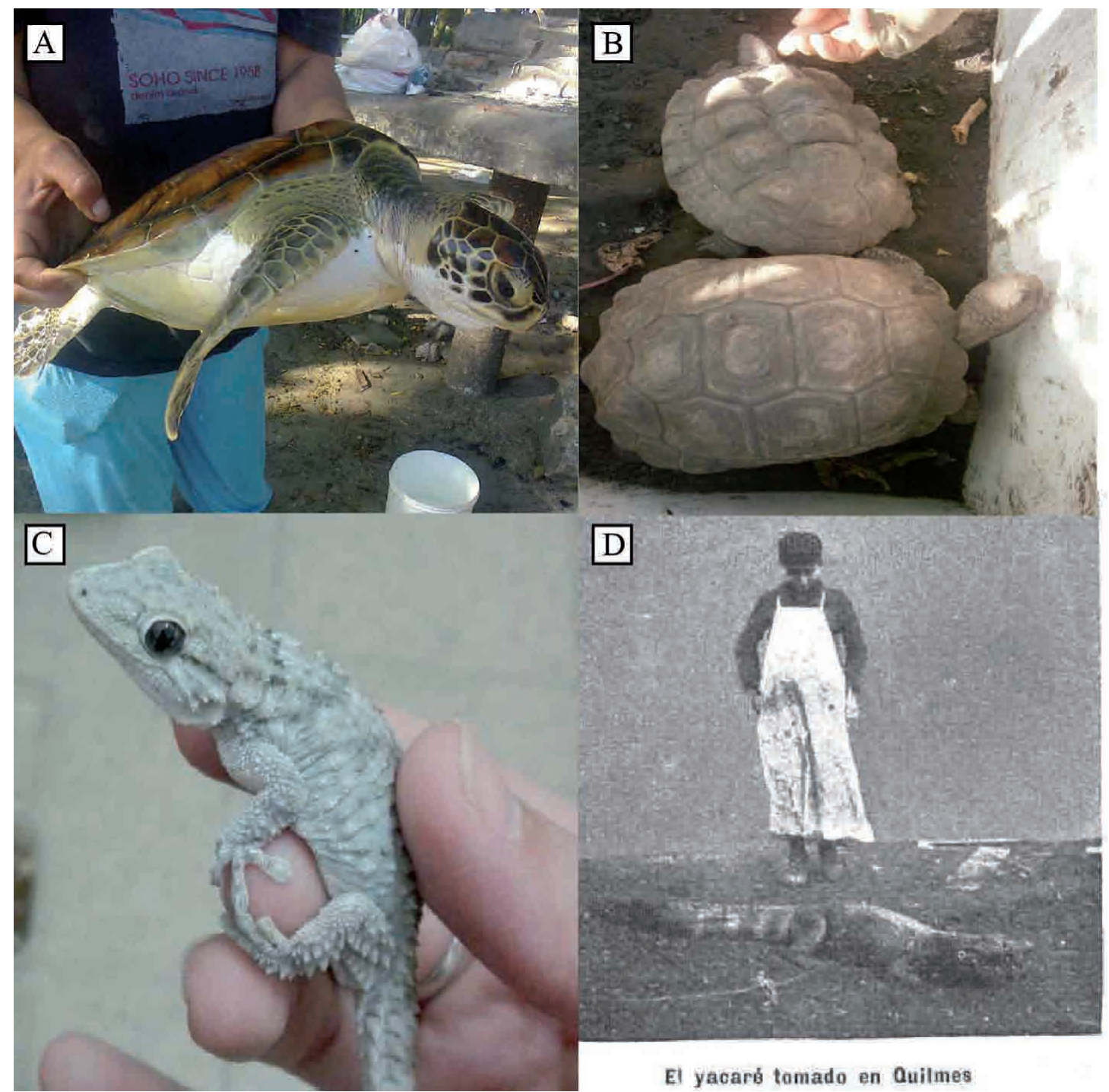

Figura 2. A, Chelonia mydas; B, Chelonoidis chilensis; C, Tarentola mauritanica; D, fotografía del yacaré capturado en el partido de Quilmes luego de las inundaciones de 1905 (Anónimo, 1905).

Se destaca el registro de la tortuga marina amenazada Chelonia mydas. Sobre la costa del Río de la Plata en la RMSMQ se hallaron dos ejemplares de esta especie a fines de noviembre del 2012 (Fig. 2A). Se han registrado varios casos en donde esta tortuga ingresa por el Río de la Plata y se le las encuentra en sus playas (Williams \& Kacoliris, 2012). Los ejemplares hallados medían aproximadamente $40 \mathrm{~cm}$ de longitud, lo cual corresponde a individuos jóvenes. Al igual que en el caso expuesto en este trabajo, los registros de dicha especie en el área corresponden a ejemplares juveniles (Chebez, Waller, Richard, 2008a). Se cuenta con dos especies exóticas de reptiles. Se obtuvo un registro incidental de Chelonoidis chilensis, una tortuga terrestre amenazada, cuya distribución natural es la Provincia fitogeográfica Chaqueña y la Provincia del Monte (Chebez, Waller, Richard, 2008b). Su aparición en la costa de la RMSMQ se produjo probablemente por la liberación o escape accidental de ejemplares capturados para mascotismo, razón por la cual se la considera como especie introducida casual (Fig. 2 B). La segunda especie, Tarentola mauritanica, se ha 
naturalizado hace algunas décadas en los ambientes urbanos de las grandes ciudades de la región (Baldo, García, Borteiro, Brusquetti, Prigioni, 2008). Se registró esta especie en las barrancas de Bernal dentro de un hogar y en una terraza (Fig. 2 C).

\section{DISCUSIÓN}

Los valores de riqueza hallados en los partidos de Avellaneda y Quilmes (13 anfibios y 18 reptiles nativos) son similares a los de la cercana Reserva Natural Punta Lara. Gran parte de las especies del área de estudio son representantes del dominio biogeográfico Paranaense (e.g., Phrynops hilarii, Thamnodynastes hypoconia, Helicops infrataeniatus, Scinax berthae, Scinax granulatus), que habitan los humedales de los ríos Paraná y Uruguay extendiendo su distribución geográfica hacia el sur hasta el Río de la Plata. La fauna del Río de la Plata es un nexo entre aquella proveniente de la región subtropical y la propia de la llanura Pampeana (Ringuelet, 1955; 1981). En base a la distribución geográfica de plantas trepadoras y epífitas, se planteó que la zona de estudio pertenecería a la provincia Paranaense (Guerrero et al., 2018), lo cual encuentra soporte con la distribución de estos componentes de la fauna de reptiles y anfibios del área de estudio.

La distribución geográfica de Phrynops hilarii, una de las tortugas registradas durante este trabajo, se ha extendido hacia el sur en las últimas décadas (Derocco, Alcalde, Rosset, 2005; Guerrero \& Agnolin, 2016). En el área de estudio se registró por primera vez en el año 2016, luego de la aparición de camalotales (comunidades vegetales flotantes dominadas por pontederiáceas) en la zona de estudio (Guerrero et al., 2017). La RMSMQ aloja numerosas especies de plantas y animales que han avanzado hacia el sur en tiempos recientes (Guerrero et al., 2012; Dosil et al., 2018), como es el caso de Phrynops hilarii. Por esta razón, se planteó que esta área protegida puede estar funcionando como stepping-stone entre áreas naturales del norte y del sur para especies que «reajustan» su distribución geográfica debido al cambio climático en la región (Guerrero et al., 2012). El Sistema Fluvial del Plata, aporta por dispersión pasiva, elementos bióticos de la zona subtropical de Sudamérica, en donde sus grandes ríos poseen las nacientes, enriqueciendo la flora y fauna de la localidad estudiada (Giraudo et al., 2004). Los camalotales traen consigo numerosos taxones, impactando en los procesos de colonización e intercambio genético entre las poblaciones extremas de algunas especies (Guerrero et al., 2017; Achaval, 1979). Es probable que la expansión hacia el sur de esta especie sea consecuencia de estos fenómenos.

Adicionalmente, se cuenta con un registro de Caiman latirostris (Daudin, 1802) que arribó sobre camalotes a la zona costera del partido de Quilmes luego de una gran inundación (Anónimo, 1905; Fig. 2 D). Dado que no se conoce exactamente el sitio del partido en el que se fotografió y capturó el ejemplar, no se lo ha incluido en la lista, pero el registro es interesante ya que ejemplifica el poder de transporte que tienen los camalotales.

La zona de la RMSMQ se encuentra amenazada por la constante urbanización y diversas fuentes de contaminación asociadas. Las zonas lindantes han sido afectadas, 
entre otras cosas, por el desarrollo de un relleno sanitario, la contaminación industrial de la papelera Smurfit Kappa, la construcción de la Autopista Dr. R. Balbín y el crecimiento urbano de las ciudades de Bernal y Don Bosco, por lo cual la mencionada reserva es el último remanente de los ambientes naturales o semi-naturales de la localidad. El albardón costero y los bañados que abarca la RMSMQ y la costa de Avellaneda fueron utilizadas como áreas productivas por quinteros hasta la década de 1980. La mayor parte del bosque existente -si no todo- se desarrolló en las últimas décadas integrando la flora y fauna remanente de los sistemas originales con aquella que arrastra en cada creciente el Río de la Plata y las especies que introdujeron los productores locales (Guerrero et al., 2012, Hurrell, Cabanillas, Guerrero, Delucchi, 2014; Dosil et al., 2018). El resultado de esta compleja sucesión es el neoecosistema de alta riqueza de especies que se observa en la actualidad, que ha permitido en tiempos recientes el establecimiento de especies de latitudes más altas que antes no lograban colonizar el Río de la Plata (Guerrero et al., 2012).

En localidades cercanas al área de estudio se citaron otras 21 especies de anfibios y reptiles. Posiblemente algunas de estas especies puedan estar presentes en la zona y no fueron halladas debido a que no se pudo visitar el área de estudio en un horario de muestreo apropiado para oír ciertos anfibios. Sin embargo, se debe remarcar que muchas de estas especies son características de distintos tipos de pastizal. En el área de estudio, los pastizales naturales fueron completamente destruidos, y los únicos pastizales que se observan en la actualidad han crecido (secundariamente) en terraplenes de caminos y sobre el relleno sanitario conformando neoecosistemas. Es por ello, que otra hipótesis para explicar su ausencia en nuestro muestreo puede ser que las especies de pastizal han sido negativamente afectadas por diversos factores antrópicos. Varias especies de anfibios y reptiles que en la actualidad son poco frecuentes en la región, como Tomodon ocellatus y Ceratophrys ornata, son sensibles a la destrucción de los pastizales nativos (Giraudo et al., 2012; Vaira et al., 2012). Probablemente estas especies se hayan extinguido de la altamente urbanizada región metropolitana de la Ciudad Autónoma de Buenos Aires, al igual que Melanophryniscus montevidensis (Agnolin \& Guerrero, 2017). Se puede plantear que los ecosistemas de la zona costera inmediata al Río de la Plata en Avellaneda y Quilmes han sufrido perturbaciones antrópicas compatibles con el grado de resiliencia de sus comunidades, ya que muestran una recuperación rápida de la taxocenosis de anfibios y reptiles; pero como contraparte, la actividad antrópica sobre la barranca y la terraza alta implicó la completa desaparición de los pastizales y bosques xeromorfos originales, degradando de manera irreversible la composición de su fauna.

\section{AGRADECIMIENTOS}

Los autores agradecen a la Asamblea Vecinal «No a la Entrega de la Costa», a A. Vasconcelos, D. Daglio, A. Victorero, C. Guillén, R. Díaz, N. Lazarte, E. Montalibet, P. Carrión y J.M. Sagan por colaborar en los trabajos de campo, a M. Rojas, a L. Sancho y la E.G.B. 52 de Avellaneda, a la familia Guerrero por capturar y fotografiar algunos materiales, a Walter Prado por su contribución en los muestreos durante el 
fenómeno de aparición de camalotes en la costa del Río de la Plata, a F. Palacio por sus consejos sobre la publicación del manuscrito, y a L. Alcalde, D. Di Pietro, J. D. Williams y a P. Knight por su atención en la colección herpetológica del Museo de La Plata y colaboración en la identificación de los especímenes. Agradecemos también a los revisores de Acta Zoológica Lilloana que contribuyeron a enriquecer el manuscrito original.

\section{FINANCIAMIENTO}

No se contó con financiamiento externo.

\section{PARTICIPACIÓN}

Todos los autores participaron del relevamiento. E. L. Guerrero dirigió los relevamientos entre 2005 y 2014 y F. Suazo Lara desde 2014 hasta 2017. E. L. Guerrero, L. Lerzo, G. Reuter, F. Suazo Lara, D. Vera y A.B. Tenorio escribieron el manuscrito. E. L. Guerrero, L. Lerzo y G. Reuter confeccionaron las tablas y E. L. Guerrero confeccionó las figuras.

\section{CONFLICTO DE INTERÉS}

No existen conflictos de intereses.

\section{LITERATURA CITADA / BIBLIOGRAPHY}

Anónimo (1905). El Chaco en Buenos Aires. Semanario Caras y Caretas, 8 (353), 56. Disponible en http://hemerotecadigital.bne.es/issue.vm?id=0004200774\&sear $\mathrm{ch}=$ \&lang $=\mathrm{es}$

Abdala, C. S., Acosta, J. L., Acosta, J. C., Álvarez, B. B., Arias, F., Avila, L. J., Breitman, M. F., Cabrera, M. R., Cairo, S., Corbalán, V., Hernando, A., Ibargüengoytía, N. R., Kacoliris, F., Laspiur, A., Montero, R., Morando, M., Pelegrin, N., Hernán, C., Pérez, F., Quinteros, A.S., Semhan, R. V., Tedesco, M. E.,Vega, L., Zalba, S. M. (2012). Categorización del estado de conservación de las lagartijas y anfisbenas de la República Argentina. Cuadernos de herpetología, 26 (supl. 1), 215-302.

Agnolin, F., Guerrero, E. L. (2017). Local extinction of Melanophryniscus montevidensis (Anura: Bufonidae) in the Argentine Pampas. Check List, 13, 11-15.

Agostini, M. A., Saibene, P., Barraso, D. A. (2012). Anfibios de la Reserva Natural de Punta Lara. En Inventario de los vertebrados de la Reserva Natural Punta Lara, provincia de Buenos Aires, Argentina (71-81). Ciudad Autónoma de Buenos Aires: Monografía de Aves Argentinas $N^{\circ} 8$. 
Agostini, M. G., Saibene, P. E., Roesler, C. I., \& Bilenca, D. N. (2016). Amphibians of northwestern Buenos Aires province, Argentina: checklist, range extensions and comments on conservation. Check List, 12 (6), 1-10.

Barrio, A. (1962). Los Hylidae de Punta Lara, Provincia de Buenos Aires. Physis, 23 (65), 129-142.

Baldo, D., García, J., Borteiro, C., Brusquetti, F., \& Prigioni, C. (2008). Reptilia, Gekkonidae, Hemidactylus mabouia, Tarentola mauritanica: Distribution extension and anthropogenic dispersal. Check List, 4, 434.

Cabanillas, P. A., Barral, L. M., Guerrero, E. L, Chimento, N. R. (2016). Categorización y valoración del estado de conservación de una sección de bosque de la ribera de Quilmes y Avellaneda (Buenos Aires, Argentina). Historia Natural, tercera serie, 6 (1), 83-109.

Cabrera, A. L., Dawson, G. (1944). La selva marginal de Punta Lara en la ribera argentina del Río de la Plata. Revista del Museo de La Plata, 22, 1-382.

Chebez, J. C., Waller, T., Richard, E., (2008a). «Tortuga Verde». En Los que se van. Fauna argentina amenazada tomo 1 (204-208). Ciudad Autónoma de Buenos Aires: Albatros.

Chebez, J. C., Waller, T., Richard, E., (2008 b). «Tortuga Terrestre Común». En Los que se van. Fauna argentina amenazada tomo 1 (219-226). Ciudad Autónoma de Buenos Aires: Albatros.

Chebez, J. C., Gasparri, B., Athor, J. (2012). Las reservas y espacios verdes urbanos. Historia y actualidad. En Buenos Aires, la historia de su paisaje natural (390409). Ciudad Autónoma de Buenos Aires: Fundación de Historia Natural Félix de Azara.

Daglio, D., Suazo Lara, F., Lazarte, N. y Lachowicz, C. (2014). Resultados preliminares del relevamiento de la malacofauna de la Reserva Municipal «Selva marginal quilmeña» (Buenos Aires, Argentina). III Congreso Uruguayo de Zoología. Libro de Resúmenes: 186.

Derocco, N. N., Alcalde L., Rosset, S. D. (2005). Ampliación de la distribución de Phrynops hilarii (Pleurodira: Chelidae) en Argentina. Cuadernos de Herpetología, 19 (1), 63.

Di Pietro, D. O. (2016). Historia natural y ecología de los ofidios (Reptilia: Serpentes) de las sierras australes de la provincia de Buenos Aires, Argentina (Tesis Doctoral, Facultad de Ciencias Naturales y Museo, Universidad Nacional de La Plata).

Dosil Hiriart, F., Cabanillas, P., Apodaca, M. J., Benedictto, M., Barral, L., Guerrero, E. L. (2018). Listado comentado de las plantas vasculares trepadoras y epífitas de la costa rioplatense del partido de Quilmes (Buenos Aires, Argentina). Boletín de la Sociedad Argentina de Botánica, 53 (1), 103-113.

Gallardo, J. M. (1974). Anfibios de los Alrededores de Buenos Aires. Buenos Aires: EUDEBA.

Gallardo, J. M. (1977). Reptiles de los Alrededores de Buenos Aires. Buenos Aires: EUDEBA.

Gallardo, J. M. (1987). Anfibios y Reptiles del Partido de Magdalena (Provincia de Buenos Aires). Buenos Aires: Artes Gráficas Rioplatense. 
Giraudo, A. R. (2001). La diversidad de serpientes de la Selva Paranaense y del Chaco Húmedo: Taxonomía, biogeografía y conservación. Buenos Aires: L.O.L.A.

Giraudo, A. R., \& Arzamendia, V. (2004). ¿Son las planicies fluviales de la Cuenca del Plata, corredores de biodiversidad? Los vertebrados amniotas como ejemplo. En Humedales de Iberoamérica (157-170). La Habana: CYTED.

Giraudo, A. R., Arzamendia, V., Bellini, G. P., Bessa, C. A., Calamante, C. C., Cardozo, G., Chiaraviglio, M., Costanzo, M. B., Etchepare, E. G., Di Cola, V., Di Pietro, D. O., Kretzschmar, S., Palomas, S., Nenda, S., Rivera, P. C., Rodríguez, M. E., Scrocchi, G. J., Williams, J. D. (2012). Categorización del estado de conservación de las serpientes de la República Argentina. Cuadernos de herpetología, 26 (supl.1), 303-374.

Godoy, I., Suazo Lara, F., Guerrero, E. L., Rivero P., González, B., Alegre, M., Godoy, A., Kain, C., Sesto, F., Chimento, N. R. (2012). Relevamiento biótico de la costa rioplatense de los partidos de Quilmes y Avellaneda (Buenos Aires, Argentina). Parte II: Aves. Historia Natural, tercera serie, 2, 57-94.

Guerrero, E. L., Suazo Lara, F., Ortiz, N. R., Buet Constantino, F., Simon, P. (2012). Relevamiento biótico de la costa rioplatense de los partidos de Quilmes y Avellaneda (Buenos Aires, Argentina). Parte I: aspectos ambientales, botánicos y fauna de Opiliones (Arachnida), Mygalomorphae, (Arachnida) y Chilopoda (Myriapoda). Historia Natural, tercera serie, 2, 31-56.

Guerrero, E. L., Agnolin F. L. (2016). Recent changes in plant and animal distribution in the southern extreme of the Paranaense biogeographical province (northeastern Buenos Aires province, Argentina): Ecological responses to climate change? Revista del Museo Argentino de Ciencias Naturales, nueva serie, 18 (1), 9-30.

Guerrero, E. L., Agnolin, F. L., Grilli, P., Suazo Lara, F. A., Boné, E., Tenorio, A. B., Derguy, M., Lucero, S., Chimento Ortíz, N. R., Milat, J. A., Nenda, S., Benedicto, M., Montalibet, E., Olmos, M., Barrasso, D., Apodaca, M. J. (2017). Inventario de la fauna transportada por balsas de vegetación flotante en el sistema fluvial del Río de la Plata. Revista del Museo Argentino de Ciencias Naturales, nueva serie, 19 (2), 177-183.

Guerrero, E. L., Apodaca, M. J., Dosil-Hiriart, F. D., Cabanillas, P. A. (2018). Análisis biogeográfico de los humedales del sistema fluvial del Río de la Plata basado en plantas trepadoras y epífitas. Revista Mexicana de Biodiversidad, 89 (4), 1190-1200.

Heyer, R., Donnelly, M. A., Foster, M., Mcdiarmid, R. (2014). Measuring and monitoring biological diversity: standard methods for amphibians. Washington D.C.: Smithsonian Institution Press.

Hurrell, J. A., Cabanillas, P., Guerrero, E. L. y Delucchi, G. (2014). Naturalización y etnobotánica de Vitislabrusca L. (Vitaceae) en la región rioplatense, Argentina. Revista del Museo Argentino de Ciencias Naturales, nueva serie, 16 (1), 13-18.

Kacoliris, F., Horlent, N., Williams, J. (2006). Herpetofauna, coastal dunes, Buenos Aires province, Argentina. Check List, 2, 15. 
Koslowsky, J. (1896). Reptiles y batracios de la Sierra de la Ventana (Provincia de Buenos Aires). Revista del Museo de La Plata, 7, 149-156.

Krebs, C. 2008. The Ecological World View. CSIRO Publishing, Colinwood, Australia. $574 \mathrm{pp}$.

Matteucci, S. D., Falcón, M. (2012). Efectos de la urbanización sobre la biodiversidad. En Buenos Aires, la historia de su paisaje natural (272-291). Ciudad Autónoma de Buenos Aires: Fundación de Historia Natural Félix de Azara.

Montalibet, E. S., Rojas, M. J., Vera, D. G., Jiménez Arevalo, N., Retali, D. F., Victorero, F. A., Benitez Aquino, M. A., Bogado, N. R., Dosil Hiriart, F., Rodríguez Lando, R., Retali, J. M., Guerrero, E. L., Peralta, L. (2015). Proyecto de relevamiento de Araneomorphae (Arachnida, Araneae) de la costa rioplatense de los partidos de Quilmes y Avellaneda (Buenos Aires, Argentina): las arañas como herramientas para la conservación. IV Encuentro de Jóvenes Aracnólogos Argentinos y X Encuentro Biólogos en Red. Mar del Plata, Argentina. Libro de resúmenes: 33 .

Montalibet E. S., Sosa M. A., Rozadilla S., Lazarte N. y Irazoqui F. M. (2016). Contribución al conocimiento de las aves presentes en la costa rioplatense de los partidos de Quilmes y Avellaneda (Buenos Aires, Argentina). Revista del $\mathrm{Mu}$ seo de La Plata, 1 (1), 29-38.

Pereira J., Haene E. (2003) Anfibios de la reserva Natural Otamendi. En Fauna de Otamendi. Inventario de los animales vertebrados de la Reserva Natural Otamendi, Campana, Buenos Aires, Argentina (29-36). Buenos Aires: Aves Argentinas/Asociación Ornitológica del Plata, temas de naturaleza y conservación 3.

Prado, W. S., Waller, T., Albareda, D. A., Cabrera, M. R., Etchepare, E. G., Giraudo, A. R., Gonzalez-Carman, V., Prosdocimis, L., Richard, E. (2012). Categorización del estado de conservación de las tortugas de la República Argentina. Cuadernos de herpetología, 26 (supl. 1), 259-271.

Ringuelet, R. A. (1955). Panorama zoogeográfico de la Provincia de Buenos Aires. Notas del Museo de La Plata, Zoología, 18, 1-15.

Ringuelet, R. A. (1981). El ecotono faunístico Subtropical-Pampásico y sus cambios históricos. En Symposia, VI Jornadas Argentinas de Zoología (75-80). La Plata: Ramos Americana Editora.

Roesler, I., Agostini, M. G., De Pino, E., Carricart, J. P., (2012). Conservación de la Reserva Natural Punta Lara. En Inventario de los vertebrados de la Reserva Natural Punta Lara, provincia de Buenos Aires, Argentina (173-183). Ciudad Autónoma de Buenos Aires: Monografía de Aves Argentina No8.

Rojas, M., Montalibet, E. S., Guerreo, E. L. (2016). Comparación de metodologías aplicadas en el muestreo de Opiliones (Arachnida) en la Reserva Natural Selva Marginal Quilmeña (Quilmes, Buenos Aires). I Jornadas Argentinas de Aracnología y V Encuentro de Jóvenes Aracnólogos Argentinos. Libro de resúmenes: 36 .

Saibene, P., Cajade, R., Pagano, L. G., Herrero, R. (2012). Reptiles de la Reserva Natural Punta Lara. En Inventario de los vertebrados de la Reserva Natural 
Punta Lara, provincia de Buenos Aires, Argentina (83-96). Ciudad Autónoma de Buenos Aires: Monografía de Aves Argentinas $\mathrm{N}^{\circ} 8$.

Sosa, M.A., Rozadilla, S., Lazarte, N., Irazoqui, F. M. y Montalibet, E. (2015). Nuevos registros de Aves para la costa rioplatense de los partidos de Quilmes y Avellaneda (Buenos Aires, Argentina). XVI Reunión Argentina de Ornitología, Libro de Resúmenes: 153.

Vaira, M., Akmentins, M. S., Attademo, A. M., Baldo, J. D., Barrasso, D. A., Barrionuevo, J. S., Cespedez, J., Blotto, A. B., Cairo, S., Cajade, R., Céspedez, J., Corbalán, V., Chilote, P., Duré, M., Falcione, C., Ferraro, D., Gutiérrez, R. F., Ingaramo, M., Junges C., Lajmanovich, R., Lescano, J. N., Marangoni F., Martinazzo L., Marti, R., Moreno, L., Natale, G. S., Pérez Iglesias, J. M., Peltzer, P., Quiroga, L., Rosset, L., Sanabria, E., Sánchez, L., Schaefer, E., Úbeda, C., Zaracho, V. (2012).Categorización del estado de conservación de los anfibios de la República Argentina. Cuadernos de herpetología, 26 (supl. 1), 131-159.

Vega, L. E., Bellagamba, P. J. (1990). Lista comentada de la herpetofauna de las sierras de Balcarce y Mar del Plata, Buenos Aires, Argentina. Cuadernos de Herpetología, 5 (2), 10-14.

Williams, J. D. (1991) Anfibios y Reptiles. Situación Ambiental de la provincia de Buenos Aires. CIC, 1 (4), 1-21.

Williams, J. D. (1992) Nueva localidad para Chthonerpeton indistinctum (Reinhardt y Lutken, 1861), en la Provincia de Buenos Aires, Argentina. (Amphibia: Gymnophiona). Neotropica, 38 (99), 58.

Williams, J. D., Kacoliris F. P. (2012). Los paisajes perdidos de Buenos Aires. Anfibios y Reptiles. En Buenos Aires, la historia de su paisaje natural (215-232). Ciudad Autónoma de Buenos Aires: Fundación de Historia Natural Félix de Azara.

\section{Apéndice}

Materiales de referencia del área de estudio depositados en la colección de herpetología del Museo de La Plata:

- Dendropsophus nanus (Boulenger, 1889): MLP 5879.

- Pseudis minuta (Günther, 1858): MLP 5878.

- Pseudis limellum (Cope, 1862): MLP 5891, MLP 5892.

- Scinax berthae (Barrio, 1962): MLP 5888.

- Scinax granulatus (Peters, 1871): MLP 5889, MLP 5890.

- Scinax squalirostris (Lutz, 1925): MLP 5880.

- Leptodactylus latinasus (Jiménez de la Espada, 1875): MLP 5139, MLP 5326, MLP 5874.

- Leptodactylus latrans (Steffen, 1815): MLP 5137, MLP 5875, MLP 5877, MLP 5886.

- Pseudopaludicola falcipes (Hensel, 1867): MLP 5881. 
- Rhinella fernandezae (Gallardo, 1957): MLP 5136, MLP 5138, MLP 5328, MLP 5873.

- Amphisbaena darwinii (Duméril \& Bibron, 1839): MLP 6557, MLP 6558.

- Cercosaura schreibersii (Wiegmann, 1834): MLP 6393.

- Epictia albipuncta (Burmeister, 1861): MLP 6684.

- Helicops infrataeniatus (Jan, 1865): MLP 6588, MLP 6589, MLP 6591.

- Helicops leopardinus (Schlegel, 1837): MLP 6560.

- Paraphimophis rusticus (Cope, 1878): MLP 6586, MLP 5961.

- Thamnodynastes strigatus (Günther, 1858): MLP 6583.

- Xenodon dorbignyi (Bibron, 1854): MLP 6585.

- Erythrolamprus semiaureus (Cope, 1862): MLP 6587, MLP 6036.

- Erythrolamprus poecilogyrus (Wied-neuwied, 1825): MLP 6392. 